\section{Elastic drug delivery: could treatments be triggered by patient movement?}

\author{
"The research and development of patients' movement-controlled \\ drug delivery systems hold promise in improving patients' compliance \\ by providing a self-directed and on-demand treatment."
}

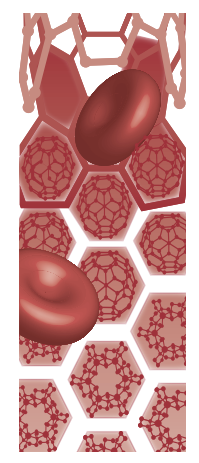

\author{
Yuqi Zhang \\ Joint Department of Biomedical \\ Engineering, University of North \\ Carolina at Chapel Hill \& North \\ Carolina State University, Raleigh, \\ NC 27695, USA
}

\section{Jicheng $\mathrm{Yu}$}

Joint Department of Biomedical Engineering, University of North Carolina at Chapel Hill \& North Carolina State University, Raleigh, NC 27695, USA tic efficacy, reduce side effects and prolong action time, vast efforts have been dedicated to the development of on-demand, precise drug release. In light of this, numerous stimuli-responsive designs have been exploited, including external triggers like mechanical force, temperature, light, ultrasound, electric current and magnetic field as well as internal factors like $\mathrm{pH}$, redox, enzymes, ATP and hypoxia [3-8]. Compared with other stimuliresponsive designs, the macroscopic mechanical force-mediated approach, as one of the most promising strategies, possesses several advantages. It can be generated on-demand during the patients' daily movement, such as tension in bone joints, tendons and muscles, or compression in cartilage and bones. Therefore, a self-administrated therapy can be readily achieved without requirement of additional instrumentations. In addition, in contrast to the inaccurate internal factors due to the complicated physiological environment, the degree of stretch or compression is more conveniently controlled by the patient themselves, leading to a precise dosage-, spatial- and temporal-controllable administration of drug release.

Physical deformation of drug carriers supported on an elastomer substrate caused by stretch or compression is one of the most important strategies for mechanical forcetriggered release. Mooney group designed a compression-responsive system for controlled release of growth factor [9]. Inspired by the natural extracellular matrices, they developed a hydrogel with reversible binding of drug as synthetic extracelluar matrices. The physical-loaded hydrogel could respond to repeated compression stimulus and as a result released free drug. Afterward, the matrices could be refilled by free drug during relaxation via dissociation of previously bound drug. Using VEGF as a model drug in in vivo studies, they demonstrated that the implanted hydrogels allowed an increase in VEGF concentration near implantation site as applying mechanical signals, subsequently leading to a local enhanced vascularization. In another case, Jeong group developed a strain-sensitive patch consisting of arrays of microcapsules onto a rubbery substrate for drug release [10]. When stretch was applied to the elastomer substrate, the volume of the stretchable microcapsules encapsulating cargoes decreased accordingly with the substrate, then pumping out the preloaded molecules. Under different degrees of mechanical stretching, the release rate and amount of cargoes could be adjusted. This patch has the potential to respond to body motions, even to the mechanical stretching of organs, muscles and tendons when it is implanted into body.

We have recently developed a multipurpose wearable, tensile strain-triggered

\section{Yong Zhu}

Department of Mechanical

\& Aerospace Engineering, North Carolina State University, Raleigh, NC 27695, USA

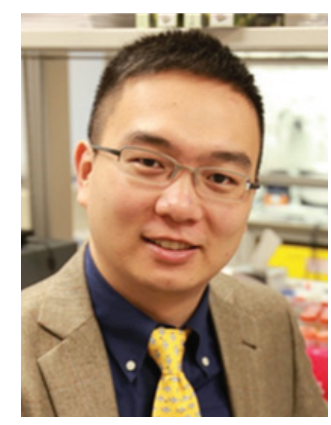

\section{Zhen Gu}

Author for correspondence: Joint Department of Biomedical Engineering, University of North Carolina at Chapel Hill \& North Carolina State University, Raleigh, NC 27695, USA

Tel.: +1 9195157944

zgu@email.unc.edu 
drug delivery device, which comprised of a stretchable elastomer and microgel depots containing drug-loaded nanoparticles [11]. The drugs can be continuously released from the nanoparticles and temporarily stored in the microgels. When applying a tensile strain, the drugs were released from microdepots due to the enlarged surface area for diffusion and Poisson's ratio-induced compression toward the microgels. Therefore, a sustained drug release can be conveniently achieved by daily body motion, while a pulsatile release is able to be controlled through intentional administration. We demonstrated that this device could be simply attached to a finger joint, and stretched to trigger the drug release when the finger is flexed for multiple cycles, which allowed patients to control the dose and release timing of antibacterial drug on their own.

Furthermore, we integrated this stretch-sensitive device with a microneedle array patch for on-demand transcutaneous insulin delivery [3], which allowed the blood glucose level of mice to decline quickly to a normoglycemic range within $0.5 \mathrm{~h}$. Meanwhile, the obvious pulsatile and continuous reduction in blood glucose level were observed when applying a strain with an interval of $4 \mathrm{~h}$. Based on this technology, the diabetic patients can easily maintain normoglycemia through simple joint movement instead of a traditional painful insulin injection. This skin-mountable device can be further extended for anti-inflammatory, anti-infective drug or painkiller delivery. More importantly, this facile strategy allows immediate medical treatment in emergency situation by patient's simple body movement.

Besides the direct drug release via changing diffusion area or pumping out caused by physical deformation, tension or compression can also generate energy to change the physical properties of drug carriers. For example, Pioletti group exploited dissipation properties of hydrogel as an internal heat source to trigger the thermal-sensitive drug release instead of additional external heat source [12]. Self heating was quickly produced after 5 min cyclic mechanical loading. The increased temperature further caused the shrinkage of thermal-responsive nanoparticles entrapped in the hydrogel and subsequent drug release.

In addition, mechanical stretch or compression is able to tune the molecular conformation and intermolecular interaction between host molecule and guest molecule, resulting in a force-triggered drug release [13,14]. Based on this phenomenon, Ariga group reported a mechanically controlled monolayer formed by a steroid cyclophane molecule with a cyclic core linked to four steroid moieties via the flexible L-lysine spacer [13]. The applied compression could lead to a cavity-forming conformation of the cyclophane. Therefore, the hydrophobic model drug was easily trapped in this hydrophobic cavity. In contrast, expansion of the monolayer could release the encapsulated drug through the molecular transformation from cavity to planarity. Similarly, they developed a mechanical stimulus-activated $\beta$-cyclodextrin $(\mathrm{CyD})$-crosslinked alginate gel [15]. As applying mild mechanical compression, the model drug ondansetron, the entrapped guest, could be released from the host $\mathrm{CyD}$ moieties, due to the change in inclusion ability of $\mathrm{CyD}$. The host-guest interactions dominated by van der Waals interactions and hydrogen bonds in a gel matrix can be more easily broken than covalent bonds, which provide a convenient on-demand administration of medicines operated intentionally by the patient.

The research and development of patients' movement-controlled drug delivery systems hold promise in improving patients' compliance by providing a self-directed and on-demand treatment. Nonetheless, there are still many remaining challenges for clinical development. For example, the current systems cannot precisely control the release dose of therapeutics. A fundamental study on the dynamic relationships between the phase transitions of materials and the relevant release profile should be closely investigated. Moreover, regarding the different movement extent and ability for different individuals, how to generate a personalized platform and consistently apply the mechanical trigger signal are difficult tasks ahead that need to be addressed. Integration of this device with other wearable modalities to monitor the realtime physiological signals (e.g., electrocardiograph, blood glucose levels or body temperature $[16,17]$ ) and motion signals $[18,19]$ might be able to provide feedback to guide the precise, personalized drug delivery. Last but not least, good biocompatibility and biodegradability for materials is extremely important for further translation of the elastic drug delivery system. Tailoring materials mimicking the structures and composites of natural systems offer a promising strategy $[5,20]$.

\section{Financial \& competing interests disclosure}

The authors have no relevant affiliations or financial involvement with any organization or entity with a financial interest in or financial conflict with the subject matter or materials discussed in the manuscript. This includes employment, consultancies, honoraria, stock ownership or options, expert testimony, grants or patents received or pending, or royalties.

No writing assistance was utilized in the production of this manuscript. 


\section{Reference}

1 Jiang T, Mo R, Bellotti A, Zhou J, Gu Z. Gel-liposomemediated co-delivery of anticancer membrane-associated proteins and small-molecule drugs for enhanced therapeutic efficacy. Adv. Funct. Mater. 24(16), 2295-2304 (2014).

2 Sun W, Jiang T, Lu Y, Reiff M, Mo R, Gu Z. Cocoon-like self-degradable dna nanoclew for anticancer drug delivery. J. Am. Chem. Soc. 136(42), 14722-14725 (2014).

3 Yu J, Zhang Y, Ye Y et al. Microneedle-array patches loaded with hypoxia-sensitive vesicles provide fast glucose-responsive insulin delivery. Proc. Natl Acad. Sci. USA 112(27), 8260-8265 (2015).

4 Mo R, Jiang T, Disanto R, Tai W, Gu Z. ATP-triggered anticancer drug delivery. Nat. Commun. 5, 3364 (2014).

5 Lu Y, Sun W, Gu Z. Stimuli-responsive nanomaterials for therapeutic protein delivery. J. Control. Release 194, 1-19 (2014).

6 Wu Z, Wu Y, He W, Lin X, Sun J, He Q. Self-propelled polymer-based multilayer nanorockets for transportation and drug release. Angew. Chem. Int. Ed. Engl. 52(27), 7000-7003 (2013).

7 Choi SW, Zhang Y, Xia Y. A temperature-sensitive drug release system based on phase-change materials. Angew. Chem. Int. Ed. Engl. 49(43), 7904-7908 (2010).

$8 \quad$ Zhang Y, Yin Q, Yin L, Ma L, Tang L, Cheng J. Chainshattering polymeric therapeutics with on-demand drugrelease capability. Angew. Chem. Int. Ed. Engl. 52(25), 6435-6439 (2013).

9 Lee KY, Peters MC, Anderson KW, Mooney DJ. Controlled growth factor release from synthetic extracellular matrices. Nature 408(6815), 998-1000 (2000).

10 Hyun DC, Moon GD, Park CJ, Kim BS, Xia Y, Jeong U. Strain-controlled release of molecules from arrayed microcapsules supported on an elastomer substrate. Angew. Chem. Int. Ed. Engl. 50 (3), 724-727 (2011).
11 Di J, Yao S, Ye Y et al. Stretch-triggered drug delivery from wearable elastomer films containing therapeutic depots. ACS Nano 9(9), 9407-9415 (2015).

12 Moghadam MN, Kolesov V, Vogel A, Klok H-A, Pioletti DP. Controlled release from a mechanically-stimulated thermosensitive self-heating composite hydrogel. Biomaterials 35(1), 450-455 (2014).

13 Ariga K, Terasaka Y, Sakai D, Tsuji H, Kikuchi J-I. Piezoluminescence based on molecular recognition by dynamic cavity array of steroid cyclophanes at the air-water interface. J. Am. Chem. Soc. 122(32), 7835-7836 (2000).

14 Michinobu T, Shinoda S, Nakanishi T et al. Mechanical control of enantioselectivity of amino acid recognition by cholesterol-armed cyclen monolayer at the air-water interface. J. Am. Chem. Soc. 128(45), 14478-14479 (2006).

15 Izawa H, Kawakami K, Sumita M, Tateyama Y, Hill JP, Ariga K. $\beta$-Cyclodextrin-crosslinked alginate gel for patientcontrolled drug delivery systems: regulation of host-guest interactions with mechanical stimuli. J. Mater. Chem. B 1(16), 2155-2161 (2013).

16 Myers AC, Huang H, Zhu Y. Wearable silver nanowire dry electrodes for electrophysiological sensing. RSC $A d v$. 5(15), 11627-11632 (2015).

17 Kim D-H, Lu N, Ma R et al. Epidermal electronics. Science 333(6044), 838-843 (2011).

18 Yao S, Zhu Y. Wearable multifunctional sensors using printed stretchable conductors made of silver nanowires. Nanoscale 6(4), 2345-2352 (2014).

19 Yao S, Zhu Y. Nanomaterial-enabled stretchable conductors: strategies, materials and devices. Adv. Mater. 27(9), 1480-1511 (2015).

20 Mitragotri S, Anderson DG, Chen X et al. Accelerating the translation of nanomaterials in biomedicine. ACS Nano 9(7), 6644-6654 (2015). 